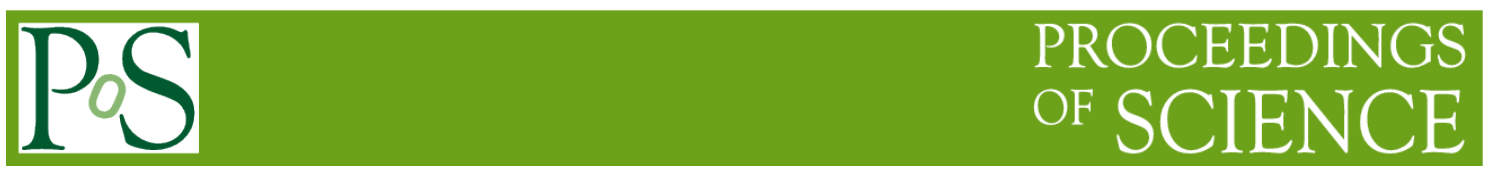

\title{
Preparing for LOFAR
}

\section{Jaap Bregman}

ASTRON

Oude Hoogeveensedijk 4, 7991 PD Dwingeloo, The Netherlands

E-mail: bregman@astron.nl

\section{Stefan Wijnholds}

ASTRON

Oude Hoogeveensedijk 4, 7991 PD Dwingeloo, The Netherlands

E-mail:wijnholds@astron.nl

\section{Michiel Brentjens}

ASTRON

Oude Hoogeveensedijk 4, 7991 PD Dwingeloo, The Netherlands

E-mail: brentjens@astron.nl

50 Years Westerbork Radio Observatory, A Continuing Journey to Discoveries and Innovations Richard Strom, Arnold van Ardenne, Steve Torchinsky (eds) 


\title{
Chapter 8 Preparing for LOFAR
}

\author{
Jaap Bregman', Stefan Wijnholds², Michiel Brentjens ${ }^{3}$
}

I

$\mathrm{n}$ a way, preparing for LOFAR was a step back in time, back to the origins of radio astronomy and back to the origins of our Universe. Radio astronomy started in 1932 when Karl Jansky discovered radio emission from the Milky Way at a wavelength of $14.6 \mathrm{~m} \mathrm{(20.5} \mathrm{MHz).} \mathrm{He} \mathrm{used} \mathrm{an} \mathrm{array} \mathrm{that} \mathrm{combined}$ the signals of eight antenna elements. This compound antenna of $30 \mathrm{~m}$ width and $4 \mathrm{~m}$ height had sufficient resolution to identify the direction of thunderstorm static, a strong interference encountered at short wave communication. Further investigations showed that the interference decreased at higher frequencies, driving radio communication to higher frequencies. Grote Reber, a radio engineer interested in astronomy, built in 1937 a reflector antenna of $9.5 \mathrm{~m}$ diameter in his backyard. Detection of celestial radiation at wavelengths of $9.1 \mathrm{~cm}(3300 \mathrm{MHz})$ and $33 \mathrm{~cm}$ (910 MHz) failed, but spring 1939 brought the first detections at $1.87 \mathrm{~m}(160 \mathrm{MHz})$. Finally, in 1944, he published the first quantitative sky map showing the Milky Way and peaks of emission in the constellations of Sagittarius, Cygnus, Cassiopeia, Canis Major, and Puppis. The radar development during wartime pushed to even higher frequencies providing more sensitive electronics and advanced dish technology that dominated radio astronomy for the second half of the $20^{\text {th }}$ century.

By 1965 the various stellar and interstellar radio emission mechanisms had been identified by their spectral and polarization properties. It was therefore decided to operate the WSRT initially at three wavelength bands at $50 \mathrm{~cm}$, $21 \mathrm{~cm}$ and $6 \mathrm{~cm}$ with accurate polarization characteristics. Operation at $92 \mathrm{~cm}$ wavelength started in 1983 and at $1.8 \mathrm{~m}$ only in 2005 !

Attempts at other institutes to image the sky at wavelengths of $4 \mathrm{~m}(74 \mathrm{MHz})$ and $8 \mathrm{~m}(38 \mathrm{MHz})$ with long arrays failed to reach the potential resolution due to ionospheric disturbances. Only the strongest sources that provided sufficient sensitivity per interferometer within an ionospheric coherence time could be properly imaged using the self-calibration technique. Even then, only a small field around the strongest source could be properly imaged and all other sources were smeared, producing a raised noise floor all over the image.

${ }^{3}$ ASTRON, The

Netherlands

2 ASTRON, Stellenbosch University,

Again, improved technology would open up the spectral window below $200 \mathrm{MHz}$ after 2005. Optical fibre technology made data transport between antenna stations affordable over distances from a few hundred meter to $50 \mathrm{~km}$ 
using bandwidths of $1 \mathrm{~Gb} / \mathrm{s}$ by 2000 . Bands of $100 \mathrm{MHz}$ could be digitized to 12-bit including all interference that would be removed later by digital processing. Only after 2005 , large computer clusters would be affordable to perform the multi-source self-calibration processing required to image all objects within a station beam and reach the theoretical noise floor. This can only be realized when the station beam is sufficiently narrow and sensitive. So, plans started in 1998 to design a Low frequency array covering 10 - $90 \mathrm{MHz}$. Even in the bands between 10 and $30 \mathrm{MHz}$, allocated to shortwave transmission, sufficient channels of only $1 \mathrm{kHz}$ wide could be selected for astronomical imaging, thanks to digital spectral Fourier filtering.

By 2003 all required technology had been demonstrated successfully with an all sky image produced with the Initial Test Station. In the meantime, it was also decided to include a second field with smaller antennas to cover the 110 $250 \mathrm{MHz}$ high band, where the Epoch of Reionization (EoR) would be visible. This EoR marks the time when the first galaxies in the Universe were formed and the first stars illuminated and ionized their surrounding neutral gas.

With LOFAR we are back at the frequencies where radio astronomy started, but now with much larger antenna fields filled with short dipole antennas having a much larger effective collecting area than their largest physical dimension and where low noise transistors contribute far less system noise than the sky itself.

The WSRT again played an important role in preparation of LOFAR's hunt to detect the EoR signal by the introduction of the Low Frequency Frontends (LFFEs) operating from $120-180 \mathrm{MHz}$ in two polarizations. See: LFFE:

Figure 1: A Low Frequency Fronten mounted on a WSRT dish. The LFFE consists of the white "handrail" style contraptions to the left of the focus box. In this image, they are in their active position. When observing at other frequencies, these are swept ou of the way using compressed air.

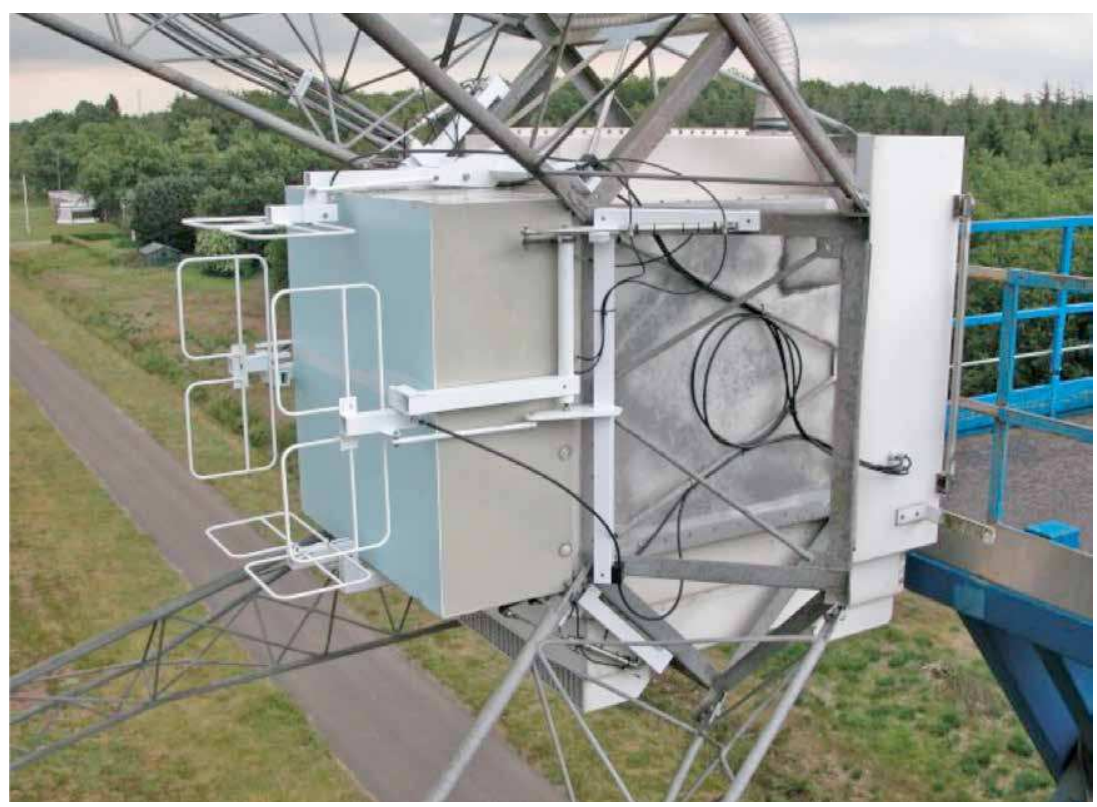

de Bruyn, Woestenburg \& van der Marel (2004). The project was lead on time on budget by Bert Woestenburg in less than two years (ASTRON LFFE Design Review Report, LFFE-00968, 2004) . As the MFFE did at higher UHF frequencies, a double dipole arrangement was used. Their performance was simulated by Marianna Ivashina and Michel Arts. The receiver design was robust in view of Interference from local transmitters. The LFFEs consisted of additional antennas placed on the focus box that could be swept in front of the MFFEs. The signals of a pair of antennas were combined and formed the first Phased Array Feed on the WSRT telescopes that provided a high sensitivity illumination pattern. Figure 1 shows an image of one of the LFFEs.

With the LFFEs, an observing campaign was conducted to select appropriate fields for the EoR experiment. After this campaign, two fields were selected (the 3C 196 field and the North Celestial Pole field) with other fields being rejected due to, for example, very complicated (but highly intriguing) Galactic foreground polarization. A side effect of this campaign was the first publication of detailed, wide-field polarimetry of the Galactic "FAN" region in the $150 \mathrm{MHz}$ band (de Bruyn, A.G. Bernardi, G; LOFAR EOR-team; "The First Deep WSRT 150 MHz Full Polarization Observations”, Proc. ASP Series, Vol. 407, 2009).

Another goal of this observing campaign was to investigate the ionospheric disturbances at the scale of the LOFAR core area with a diameter of about 2-3 km and to understand the RFI situation. The latter posed an interesting engineering challenge. Since a nearby TV transmitter, which would go out of operation only in 2006 , produced a strong signal that could not be handled by the 2-bi digitization of the DZB, additional filters had to be placed in the receiver module in the focus box. These observations were thus instrumental to gain experience in the data processing techniques that would be needed to analyze LOFAR data, in particular for techniques dealing with the radio interference at these low frequencies, and the direction dependent calibration that would be required to deal with the distortions of the received wavefront caused by the ionosphere. As such, the LFFE system played a pivotal role in understanding the challenges that LOFAR would face and allowed us to start developing solutions and software to deal with those problems well before the roll-out of LOFAR started.

With low-frequency phased array systems like LOFAR, direction dependent gain variations are not only caused by the ionosphere, but also by the varying beam shape of the receiving system while a source field is tracked on the sky. This behavior is distinctly different from the behavior of a dish beam, so there was a clear desire to properly understand this. A second experiment was therefore to combine the 14 dishes of the WSRT with a flat phased array station that used 64 prototype antennas for the LOFAR high band antenna system (HBA) system. https://www.astron.nl/r-d-laboratory/projects/wsrt-observes-lofar-highband/wsrt-observes-lofar-
highband. 
Figure 2: Pre-production prototype of a single LOFAR HBA tile at the WSRT site (excerpt from Panorama picture made by Harm-Jan Stiepel and Adriaan Renting).
Figure 3: Correlations measured on Cas A between the WSRT dishes indicated and the pre-production HBA prototype tile (image credit: Sarod Yatawatta, AJDI 29 November 2006).
Initially, the HBA beams were validated by drift scans on satellite transmissions as integration in the WSRT system turned out to be more challenging than expected. For example, a clever interface to the WSRT control software needed to be developed. This allowed us to exploit the full capabilities provided by electronic beam steering the HBA prototype in a joint observation with the WSRT dishes, while the analog receive paths had to be tuned carefully in view of the higher susceptibility of the HBA prototype to RFI. Once these technical issues were sorted, this setup was successfully used to make joint observations.

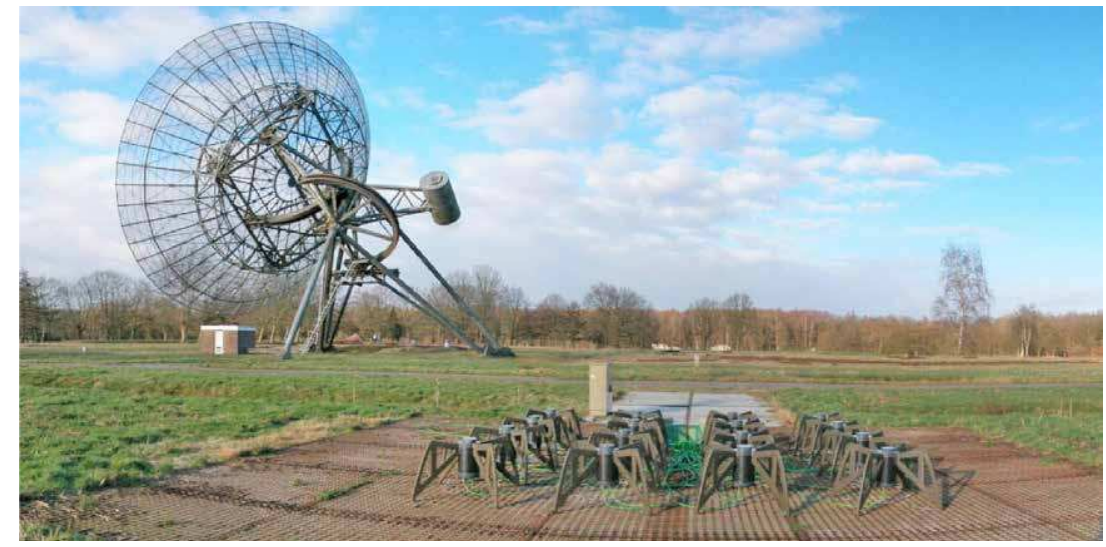

Figure 2 shows the 16 -element pre-production prototype of a single LOFAR HBA tile that succeeded the 64 -element engineering prototype after some further improvements to the designs of the HBA beamformer electronics were made. Its performance was, amongst others, assessed by a drift scan on Cassiopeia A while the tile beamformer output signals was correlated against the signals from the WSRT dishes that were tracking Cas A. Figure 3 shows the measurement results.

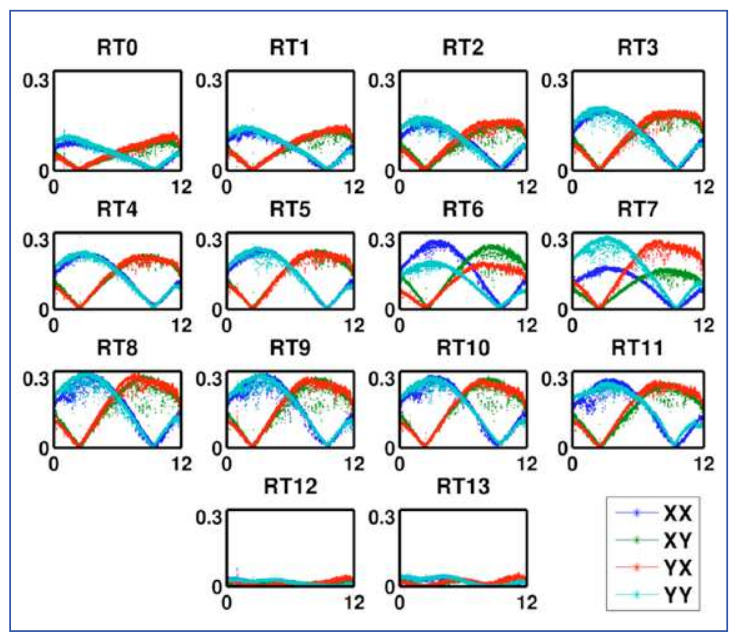

As a bonus of all these efforts, the first UltraHigh Energy Cosmic Ray event was detected, again demonstrating the serendipitous discoveries that can be made when a new receiving system is installed. 